\title{
Desenvolvimento e caracterização de filmes curativos à base de mesocarpo de Attalea speciosa Mart. ex Spreng e extrato glicólico de Aloe vera (L.) Burm. f.
}

Development and characterization of curative fillms based on Attalea speciosa Mart mesocarp. ex Spreng and glycolic extract of Aloe vera (L.) Burm. f.

Desarrollo y caracterización de películas curativas basadas en el mesocarpio de Attalea speciosa Mart. ex Spreng y extracto glicólico de Aloe vera (L.) Burm. F.

Fernanda Oliveira Sousa Araruna ORCID: https://orcid.org/0000-0002-0152-8988

Universidade Federal do Maranhão, Brasil E-mail: nandiveira@yahoo.com.br

Felipe Bastos Araruna ORCID: https://orcid.org/0000-0002-7784-6157 Universidade Federal do Maranhão, Brasil E-mail: araruna.fb@gmail.com

Luciana Patrícia Lima Alves Pereira ORCID: https://orcid.org/0000-0002-6285-4330

Universidade Federal do Maranhão, Brasil E-mail: 1lucianapatricia@yahoo.com.br

Maria Cristiane Aranha Brito

ORCID: https://orcid.org/0000-0002-6979-8773 Faculdade Maurício de Nassau São Luís, Brasil E-mail: tiane91@hotmail.com

Paulo Dyago Borges Gomes ORCID: https://orcid.org/0000-0002-5926-8939 Universidade Ceuma, Brasil E-mail: pauloborges.99@outlook.com

Danyelle Cristina Pereira Santos ORCID: https://orcid.org/0000-0002-8402-9913 Universidade Ceuma, Brasil

E-mail: danyellesantosbiomed@gmail.com

Wellyson da Cunha Araújo Firmo ORCID: https://orcid.org/0000-0002-6979-1184 Universidade Ceuma, Brasil

E-mail: well_firmo@hotmail.com Adones Almeida Rocha

ORCID: https://orcid.org/0000-0001-7282-3290 Universidade Federal do Maranhão, Brasil E-mail: adonesalmeida02@gmail.com

Klinger Antônio da França Rodrigues

ORCID: http://orcid.org/0000-0003-3904-3529 Universidade Federal do Delta do Parnaíba, Brasil E-mail: klinger.antonio@gmail.com Audirene Amorim Santana ORCID: https://orcid.org/0000-0003-3149-6822 Universidade Federal do Maranhão, Brasil E-mail: audirene.santana@ufma.br Lívio César Cunha Nunes ORCID: https://orcid.org/0000-0002-1178-7940 Universidade Federal do Piauí, Brasil E-mail: liviocesar@hotmail.com Denise Fernandes Coutinho ORCID: https://orcid.org/0000-0002-5665-9280 Universidade Federal do Maranhão, Brasil E-mail: deniseufma2014@gmail.com

\section{Resumo}

O amido de mesocarpo de babaçu (Attalea speciosa Mart. ex Spreng) e a Aloe vera (Aloe vera (L.) Burm. f.) são materiais naturais com vasta aplicação nas áreas de saúde. Neste trabalho de pesquisa foram preparados e caracterizados 
filmes casting compostos por amido de mesocarpo de babaçu suplementados com Aloe vera nas proporções de $0 \%, 5 \%$, $8 \%$ e $10 \%$ observando características físicas de espessura, umidade, solubilidades em água, permeabilidade ao vapor de água e propriedades mecânicas. Além disso, foram avaliadas a citotoxicidade da solução filmogênica em macrófagos murinos da linhagem RAW 264.7 e células de hemácias humanas. Os resultados mostram que os filmes desenvolvidos têm superfície lisa, boa maleabilidade e são autossustentados. O teor de umidade, solubilidade e permeabilidade ao vapor de água aumentaram de acordo com o aumento da concentração de Aloe vera, para as propriedades mecânicas não houve diferença significativa $(\mathrm{p}>0,05)$ entre as formulações testadas. A solução filmogênica apresentou efeito citotóxico considerado seguro para os macrófagos e hemácias humanas. Essas características demonstram um bom potencial para cobertura de feridas e reparação tecidual.

Palavras-chave: Aloe vera; Babaçu; Filmes; Reparação tecidual.

\begin{abstract}
Babassu mesocarp starch (Attalea speciosa Mart. Ex Spreng) and Aloe vera (Aloe vera (L.) Burm. f.) Are natural materials with wide application in the health fields. In this research work, casting films composed of babassu mesocarp starch supplemented with Aloe vera in the proportions of $0 \%, 5 \%, 8 \%$ and $10 \%$ were prepared and characterized, observing physical characteristics of thickness, humidity, water solubilities, vapor permeability of water and mechanical properties. In addition, the cytotoxicity of the filmogenic solution was evaluated in murine macrophages of the RAW 264.7 strain and human red blood cells. The results show that the developed films have a smooth surface, good malleability and are self-supporting. The moisture content, solubility and water vapor permeability increased according to the increase in the concentration of Aloe vera, for the mechanical properties there was no significant difference ( $p>$ 0.05 ) between the formulations tested. The filmogenic solution showed a cytotoxic effect considered safe for human macrophages and red blood cells. These characteristics demonstrate a good potential for wound coverage and tissue repair.
\end{abstract}

Keywords: Aloe vera; Babassu; Films; Tissue repair.

\title{
Resumen
}

El almidón de mesocarpio de babasú (Attalea speciosa Mart. Ex Spreng) y el Aloe vera (Aloe vera (L.) Burm. f.) son materiales naturales con amplia aplicación en el campo de la salud. En este trabajo de investigación se prepararon y caracterizaron películas de colada compuestas por almidón de mesocarpio de babasú suplementado con Aloe vera en las proporciones de $0 \%, 5 \%, 8 \%$ y $10 \%$, observando características físicas de espesor, humedad, solubilidades en agua, permeabilidad al vapor agua y propiedades mecánicas. Además, se evaluó la citotoxicidad de la solución filmógena en macrófagos murinos de la cepa RAW 264.7 y glóbulos rojos humanos. Los resultados muestran que las películas reveladas tienen una superficie lisa, buena maleabilidad y son autoportantes. El contenido de humedad, solubilidad y permeabilidad al vapor de agua aumentaron de acuerdo con el incremento en la concentración de Aloe vera, para las propiedades mecánicas no hubo diferencia significativa ( $p>0.05$ ) entre las formulaciones ensayadas. La solución filmógena mostró un efecto citotóxico considerado seguro para macrófagos humanos y glóbulos rojos. Estas características demuestran un buen potencial para cubrir heridas y reparar tejidos.

Palabras clave: Aloe vera; Babasú; Películas; Reparación de tejidos.

\section{Introdução}

A cicatrização de feridas é um processo complexo dividido em fases, classificadas como inflamatória, proliferativa e de remodelação. Durante essas etapas há uma série de eventos complexos e coordenados, que incluem quimiotaxia, fagocitose, degradação do colágeno, neocolagenase e remodelação do colágeno (Khan et al., 2013). Em geral, os estudos atuais sobre reparo tecidual envolvem principalmente o mecanismo regulador de processos de cicatrização normal e anormal e formas eficazes de promover o fechamento rápido de feridas, especialmente as crônicas (Pan et al., 2021).

Os polímeros naturais têm sido uma excelente alternativa para o tratamento de ferimentos, tendo grande destaque o amido, o qual é um polissacarídeo biocompatível e biodegradável de baixo custo e com alta disponibilidade. Este material polimérico pode atuar como espessante, estabilizador e regulador de propriedades funcionais, além de auxiliar no desenvolvimento de filmes curativos biodegradáveis e cicatrizantes, O amido de babaçu reúne todas essas características, podendo ser obtido por meio de técnicas simples (Rezler \& Poliszko, 2010; Eskandarinia et al., 2019).

O babaçu (Attalea speciosa Mart. ex Spreng.) é uma palmeira pertencente à família Arecaceae. O seu fruto é um coco composto de quatro partes, sendo o epicarpo fibroso a parte mais externa, o mesocarpo a camada intermediária e rica em amido, endocarpo a camada de madeira dura e mais interna e a amêndoa a porção mais explorada industrialmente (Teixeira, 2008; Brito, 
2015).

O mesocarpo do coco babaçu é uma biomassa subutilizada pela indústria (De Almeida et al., 2011; Maniglia et al., 2019), visto que é utilizado na maioria das vezes como suplemento alimentar, por ser rico em carboidratos e minerais, após ser extraído, pulverizado e ressuspenso em água, ademais é empregado na prática popular para solucionar problemas de saúde como: dismenorreia, constipação, obesidade, reumatismo, úlceras, doenças venosas e inflamatórias. Estudos científicos têm demonstrado várias atividades biológicas a esse mesocarpo como: anti-inflamatório (Nascimento et al., 2006), cicatrizante (Batista et al., 2006), antitumoral (Rennó et al., 2008; Fortes et al., 2009), antitrombótico (Azevedo et al., 2007) e antimicrobiano (Caetano et al., 2002).

O amido de várias fontes vegetais, tem sido descrito como matéria-prima para a produção de filmes curativos, no entanto ainda há poucos produtos no mercado (Ashraf et al., 2019).

Os curativos poliméricos representam um método eficaz para o tratamento de feridas, no entanto, para alguns tipos, como feridas infectadas, podem não ser suficiente para promover o processo de cicatrização, já que muitos desses materiais não apresentam atividade terapêutica (Pereira et al., 2013). Para resolver esta limitação, têm sido desenvolvidos curativos de polímeros naturais incorporando diferentes drogas a fim de reduzir o crescimento de microrganismos em feridas e acelerar a cicatrização (Silva et al., 2013).

Filmes curativos com efeito cicatrizante suplementados com Aloe vera tem uma boa eficácia tornando possível sua aplicação na engenharia de tecidos (Jithendra et al., 2013; Tummalapalli et al., 2016). A planta Aloe vera (Aloe vera (L.) Burm. f.), também conhecida como babosa, pertence à família Asphodelaceae (Liliaceae). A parte interna dessa planta contém um gel transparente com cerca de $99 \%$ de água, o restante é composto de acemananas, glucomananas, aminoácidos, lipídios, esteróis e vitaminas (Surjushe et al., 2008).

A Aloe vera é conhecida e usada há séculos por suas propriedades medicinais, de saúde, beleza, e cuidados com a pele. Estudos têm demonstrado que o tratamento com extratos de gel de Aloe vera resultou na cicatrização mais rápida de feridas promovendo um efeito imunoestimulante ao ativar macrófagos (Oryan et al., 2010; Pathalamuthu et al., 2019). Ela também foi descrita para retardar o envelhecimento celular, para tratar acne, psoríase, radiação ultravioleta e lesões associadas à quimioterapia, bem como para cicatrização de feridas, por se apresentar como antisséptico, antibacteriano, antiinflamatório, antioxidante, anticâncer e com efeito imunomodulador (Pathalamuthu et al., 2019; Lima et al., 2020).

As propriedades curativas se devem ao Glucomanano, um polissacarídeo rico em manose, e giberelina, que é um hormônio de crescimento. Esses compostos interagem com os receptores do fator de crescimento de fibroblasto, estimulando assim sua atividade e proliferação, o que por sua vez aumenta significativamente a síntese de colágeno após o uso de Aloe vera tópica e oral (Oryan et al., 2010).

Por ser rico em amido, cerca de 50 a $60 \%$ da sua composição, o mesocarpo de babaçu tem sido estudado como adjuvante farmacêutico e na fabricação de filmes para as mais diversas aplicações. Dessa forma, este trabalho objetiva de avaliar a utilização desse polissacarídeo como matéria-prima para a fabricação de filmes com Aloe vera que possam ser usados como curativos cicatrizantes.

É importante salientar que não foram encontrados na literatura artigos sobre o desenvolvimento de filmes à base de amido de mesocarpo de babaçu e Aloe vera, como possíveis agentes terapêuticos no tratamento de feridas.

\section{Metodologia}

Obtenção e purificação do amido de mesocarpo do babaçu (Attalea speciosa Mart. ex Spreng.)

O pó do mesocarpo foi obtido a partir da moagem do coco babaçu (Attalea speciosa Mart. ex Spreng.), coletado no município de Esperantinópolis no estado do Maranhão. O amido contido no mesocarpo foi extraído por peneiração sucessiva 
usando tamises de malhas finas com aberturas entre 0,42 e 0,125 mm. Em seguida o material foi lavado com uma solução de metabissulfito de sódio ( $5 \mathrm{~g} / \mathrm{L}$ de água), numa proporção de $1 \mathrm{~kg}$ de matéria-prima por $2 \mathrm{~L}$ de solução de metabissulfito para prevenir o escurecimento do amido, foi agitado por $10 \mathrm{~min}$, e filtrado utilizando papel de filtro com funil de Büchner em bomba de vácuo.

Do amido extraído retirou-se resíduos orgânicos fazendo lavagens sucessivas com uma solução de hidróxido de sódio $(\mathrm{NaOH})$ a 0,05 mollL e com álcool etílico absoluto, respectivamente, novamente filtrou-se e levou-se à estufa entre 30 a $45^{\circ} \mathrm{C}$ até peso constante. Metodologia adaptada de Santos, (2009).

Elaboração dos filmes casting à base de amido de mesocarpo de babaçu (Attalea speciosa Mart. ex Spreng.) e Aloe vera (L.) Burm. f.b

Os filmes foram preparados por casting a partir de uma suspensão de $4 \%(\mathrm{~m} / \mathrm{m})$ de amido de mesocarpo de babaçu em água destilada. A suspensão foi homogeneizada por 30 minutos utilizando um agitador magnético, e depois aquecida a $80^{\circ} \mathrm{C}$ por 30 minutos. Em seguida, foi adicionado o plastificante (20 g de glicerol/100 g de amido de mesocarpo de babaçu), e aquecida na mesma temperatura por mais 15 minutos em agitação. Após o resfriamento do gel foi adicionado o extrato glicólico de Aloe vera nas proporções de 5,8 e $10 \%$ e agitado abundantemente até obter uma solução homogênea.

A solução filmogênica obtida foi vertida nas placas de acrílico $(7,5 \mathrm{~g}$ por placa de $9 \mathrm{~cm})$, sendo identificadas pelas siglas: FMSAV - filme de mesocarpo sem Aloe vera; FMAV 5\% - filme de mesocarpo com 5\% de Aloe vera; FMAV 8\% - filme de mesocarpo com 8\% de Aloe vera e FMAV 10\% - filme de mesocarpo com 10\% de Aloe vera. Em seguida foi iniciado o período de secagem durante $24 \mathrm{~h}$ em estufa a $35^{\circ} \mathrm{C}$. Os filmes secos foram cortados em moldes e acondicionados em dessecador com a umidade relativa controlada em $52 \%$ e temperatura de $25^{\circ} \mathrm{C}{ }^{\circ} \mathrm{C}$ por $24 \mathrm{~h}$.

\section{Microscopia óptica}

As fotomicrografias do amido de mesocarpo purificado, solução filmogênica e filme do mesocarpo, foram realizadas com auxílio de microscópio óptico, acoplado com câmera fotográfica digital.

\section{Características dos filmes de mesocarpo de babaçu com Aloe vera}

\section{Espessura ( 8 )}

A espessura dos filmes foi determinada através da média aritmética de onze medidas aleatoriamente na superfície do filme (área $=28,26 \mathrm{~cm}^{2}$ ), usando um micrômetro externo da marca Carbografite (Capacidade: $0-25 \mathrm{~mm}$ - leitura: 0,01 mm).

\section{Conteúdo de umidade $(\omega)$}

Foi utilizado o método gravimétrico de secagem em estufa (Solar, SL-100A, Brasil) a $105{ }^{\circ} \mathrm{C}$ por $24 \mathrm{~h}$ de acordo com a metodologia $\mathrm{AOAC} \mathrm{n}^{\circ} 926.12$ (AOAC, 2007). O teor de umidade foi determinado em triplicata para cada formulação e expresso como a fração de massa após o cálculo da média.

\section{Solubilidade em água (S)}

A solubilidade foi determinada em triplicata usando a metodologia adaptada de Tapia-Blácido et al. (2011) na qual as massas determinadas para cada filme composto $\left(m_{i}\right)$ foram imersas em $50 \mathrm{~mL}$ de água destilada e agitadas constantemente em uma mesa agitadora (modelo 3545-40-EA, Termo Fisher Sci Inc., EUA) por $24 \mathrm{~h}$ à temperatura ambiente $25^{\circ} \mathrm{C}\left({ }^{ \pm} 2{ }^{\circ} \mathrm{C}\right)$. O material foi então seco em estufa (Nova Técnica, modelo 400-1ND, Brasil) a $105^{\circ} \mathrm{C}$ por $24 \mathrm{~h}$, para obtenção da massa seca $\left(m_{f}\right)$, sendo a solubilidade calculada conforme a Equação 1: 


$$
S=\left\{\frac{m_{i}(1-\omega)-m_{f}}{m_{i}(1-\omega)}\right\} \times 100
$$

Onde: $\omega$ é o teor de umidade $(\mathrm{g} / 100 \mathrm{~g}) ; \mathrm{m}_{\mathrm{i}}$ é a massa inicial do material $(\mathrm{g})$.

\section{Permeabilidade ao vapor de água (PVA)}

A permeabilidade ao vapor de água (PVA) foi determinada usando um método gravimétrico de acordo com a metodologia E96/E96M-16 (ASTM 2016). Amostras retangulares com áreas de aproximadamente $36 \mathrm{~cm}^{2}$ foram cortadas de cada filme composto e colocados em recipientes de acrílico contendo $\mathrm{CaCl}_{2}$ anidro (Êxodo, Brasil) para manter o teor de umidade dentro do recipiente em aproximadamente $0 \%$. Os compósitos foram cuidadosamente colocados no topo dos recipientes, os quais foram colocados em outro frasco contendo uma solução de $\mathrm{NaCl}$ (Synth, Brasil) na parte inferior para estabelecer uma umidade relativa de $75 \%$, obtendo-se assim, uma diferença na pressão de vapor. A taxa de aumento da massa total do filme foi obtida monitorando-se as medidas por $72 \mathrm{~h}$ para obter a permeabilidade ao vapor de água através do filme. Os valores de PVA foram obtidos usando a Equação 2:

$$
\mathrm{PVA}=\left[\frac{G \delta}{A_{e} \Delta \mathrm{P}_{a}}\right] x F
$$

Onde: $\delta$ é a espessura do filme (mm), $\mathrm{G}$ a taxa de permeabilidade à água ( $\mathrm{g} / \mathrm{dia})$, calculada a partir da regressão linear da razão de massa por tempo, $\mathrm{A}_{\mathrm{e}}$ é a área da superfície do filme exposta $\left(\mathrm{m}^{2}\right), \Delta \mathrm{P}_{\mathrm{a}}$ é a pressão parcial da água através do compósito $(\mathrm{kPa})$. F é um fator de correção que leva em consideração a resistência adicional à transferência de uma massa de vapor d'água através de um filme estagnado com ar entre a superfície da camada de $\mathrm{CaCl}_{2}$ e o filme. Pela metodologia utilizada, essa correção foi muito próxima de 1,0 e não foi considerada (da Silva Raposo et al., 2020).

\section{Propriedades mecânicas}

A resistência à tração (TS, MPa), alongamento na ruptura (E\%) e o módulo de elasticidade (EM, MPa) foram determinados diretamente para ambas as formulações em uma máquina de ensaio universal (modelo XLW (B), Labthink, EUA) à temperatura de $25^{\circ} \mathrm{C}\left( \pm 2^{\circ} \mathrm{C}\right)$ e umidade relativa de $55 \%( \pm 3 \%)$, de acordo com o método padrão D-882 da ASTM International Methods (ASTM, 2012). As espessuras de um mínimo de doze amostras de filme de $10 \mathrm{~cm} \times 2,5 \mathrm{~cm}$ foram pré-determinadas a partir da média aritmética de dez medições feitas em pontos aleatórios nos filmes compostos. Um computador foi usado para registrar as curvas de tensão-deformação e as propriedades de tração foram calculadas usando o software da máquina de ensaio universal. Uma velocidade de $1 \mathrm{~cm} / \mathrm{s}$ foi usada no teste de resistência à tração. As equações 3 e 4 são as equações para resistência à tração e alongamento na ruptura, respectivamente:

$$
\begin{aligned}
& T S=\frac{F_{m}}{A_{0}} \\
& E=\frac{l_{m}-l_{0}}{l_{0}}
\end{aligned}
$$

Onde: $F m(\mathrm{~N})$ é a força máxima antes da quebra, $A_{0}$ é a área transversal inicial do filme, $l_{m}$ é o comprimento máximo ativo e $l_{0}$ é o comprimento inicial do filme. 


\section{Efeito citotóxico em macrófagos RAW 264.7 da solução filmogênica}

Neste trabalho foram utilizados macrófagos murinos da linhagem RAW 264.7 mantidos no Laboratório de Doenças Infecciosas (LADIC) da Universidade Federal do Delta do Parnaíba (UFDPar). Foram cultivadas em meio Eagle Modificado por Dulbecco (DMEM, do inglês Dulbecco's Modified Eagle's Medium) (Sigma-Aldrich, St. Louis, EUA), suplementado a 10\% de soro fetal bovino (SFB) e penicilina-estreptomicina $10.000 \mathrm{UI} / 10 \mathrm{mg}$ (denominado DMEM completo neste trabalho) e incubados a $37^{\circ} \mathrm{C}$ em $5 \%$ de $\mathrm{CO}_{2}$.

Em placa de 96 poços foram adicionados $100 \mu \mathrm{L}$ de meio DMEM suplementado e cerca de 1 x $10^{6}$ macrófagos RAW 264.7 por poço. Essas células foram incubadas em estufa a $37^{\circ} \mathrm{C}$ e $5 \%$ de $\mathrm{CO}_{2}$ por $3 \mathrm{~h}$ para adesão celular. Após esse tempo, foram feitas duas lavagens com meio DMEM previamente aquecido a $37^{\circ} \mathrm{C}$ para retirada das células que não aderiram. Posteriormente, foram adicionados $100 \mu \mathrm{L}$ de DMEM suplementado contendo a solução-estoque da base do filme previamente diluído em DMSO e incubados a $37^{\circ} \mathrm{C}$ e $5 \%$ de $\mathrm{CO}_{2}$ por $72 \mathrm{~h}$. Ao final desse período, foram adicionados $10 \mu \mathrm{L}$ de $\mathrm{MTT}$ atingindo a concentração de $5 \mathrm{mg} / \mathrm{mL}$ e incubados por mais $4 \mathrm{~h}$ em estufa a $37^{\circ} \mathrm{C}$ com $5 \%$ de $\mathrm{CO}_{2}$. Em seguida foram adicionados $50 \mu \mathrm{L}$ de uma solução a $10 \%$ de SDS e reincubados overnight a $37^{\circ} \mathrm{C}$ com $5 \%$ de $\mathrm{CO}_{2}$ para completa dissolução do formazan. Por último, foi realizada a leitura da absorbância a $550 \mathrm{~nm}$ em leitora de placa. Os resultados foram expressos em porcentagem de viabilidade celular e em valores de concentração citotóxica para 50\% dos macrófagos $\left(\mathrm{CC}_{50}\right)$, sendo o grupo controle, realizado com meio DMEM completo a 0,5\% de DMSO, considerado como 100\% de viabilidade dos macrófagos (Rodrigues et al., 2021).

\section{Atividade hemolítica da solução filmogênica}

O teste de hemólise foi feito com hemácias obtidas de um humano voluntário e saudável (sem histórico recente de antibioticoterapia ou com antiinflamatórios e/ou doenças infecciosas ou inflamatórias três semanas antes da coleta da amostra). O estudo foi analisado e aprovado pelo Comitê de Ética em Pesquisa em Seres Humanos da Universidade CEUMA, Brasil (CEPUNICEUMA) sob o protocolo número 1.732 .522 e foi realizado de acordo com a Declaração de Helsinque 1975, revisada em 2008.

Os ensaios hemolíticos foram realizados de acordo com o método previamente descrito por Yang, Sun e Fang (2005) e Oliveira et al. (2011), modificado. Resumidamente, coletou-se o sangue e uma alíquota de $4 \mathrm{~mL}$ foi lavada três vezes, com solução salina $(0,9 \%)$. As soluções foram então centrifugadas a $3000 \mathrm{rpm}$, durante $5 \mathrm{~min}$, sendo o sobrenadante descartado. Os eritrócitos sedimentados no tubo foram diluídos em solução salina até obtenção de uma suspensão a $1 \%$. Um volume de $0,5 \mathrm{~mL}$ desta suspensão de células foi misturado a $0,5 \mathrm{~mL}$ das soluções formadoras do filme à base mesocarpo e Aloe vera nas concentrações de $0 \%, 5 \%, 8 \%$ e $10 \%$ de Aloe vera.

As misturas foram incubadas a $37^{\circ} \mathrm{C}$, com agitação contínua, durante $60 \mathrm{~min}$. As soluções foram, então, centrifugadas a 3000 rpm por $5 \mathrm{~min}$. Em seguida, a absorbância do sobrenadante foi medida a $540 \mathrm{~nm}$. As suspensões de hemácias acrescidas de solução salina e de água destilada foram, respectivamente, os controles hemolíticos, mínimo e máximo. Para eliminar a interferência das soluções formadoras do filme na absorbância, foram preparadas soluções controle, não havendo a adição da solução de hemácias. Todos os experimentos foram realizados em triplicata e expressos como porcentagem e concentração hemolítica média.

O nível de hemólise percentual foi calculado de acordo com a seguinte equação (5):

$\%$ Hemólise $=[($ At - An $) \times 100] /($ Ac - An $)$

Aqui: At é a absorbância da amostra de teste. 
An é a absorbância do controle negativo (Solução salina).

Ac é a absorbância do controle positivo (Água destilada).

\section{Análises Estatísticas}

O programa GraphPad Prism 6 foi usado para análises dos dados. As comparações estatísticas entre os grupos foram analisadas usando o teste de Tukey. Um $\mathrm{p}<0,05$ foi considerado significativo.

\section{Resultados e Discussão}

\section{Microscopia óptica}

Brito (2015) avaliou microscopicamente o pó do mesocarpo do coco babaçu, tendo sido identificados setes tipos de grãos de amido com morfologias diferentes, sendo esta caracterização útil para avaliar a autenticidade ou a origem vegetal desse produto. Os amidos de uma maneira geral quando analisados microscopicamente podem ter suas origens identificadas através da morfologia de seu grão, como de milho, arroz, mandioca. Este trabalho foi utilizado como parâmetro para a comprovação da autenticidade do amido testado (Figura 1-A). Os grãos foram ainda analisados microscopicamente após tratamento com água à temperatura de $80^{\circ} \mathrm{C}$. Na Figura 1-B, observa-se o intumescimento e a desestruturação dos grânulos de amido após a cocção à $80^{\circ} \mathrm{C}$ na solução utilizada para a fabricação do filme, a Figura 1-C demonstra a coloração do filme em tom de marrom e a presença de poucas fibras.

Figura 1. Microscopia óptica das amostras de amido de mesocarpo do coco babaçu (Attalea speciosa Mart. ex Spreng.) (A), solução de gel formador do filme (B) e filme após secagem (C) (40X)

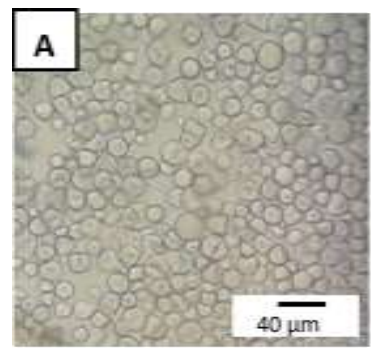

Amido

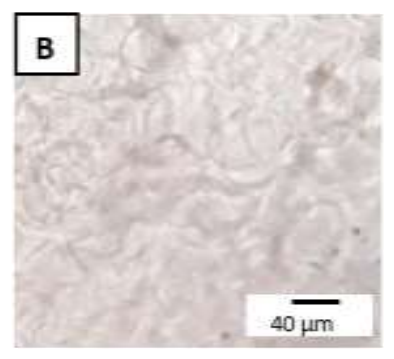

Solução filmogênica

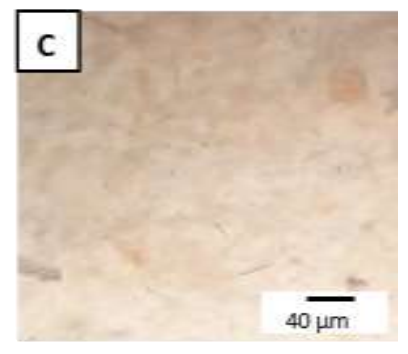

Filme seco

Fonte: Autores (2021).

Os filmes preparados à base de amido de mesocarpo de babaçu (Attalea speciosa Mart. ex Spreng.) e Aloe vera (L.) Burm. f. apresentaram superfície lisa, boa maleabilidade e foram facilmente removidos das placas demonstrando o seu potencial como filme autossustentado, conforme mostrado na Figura 2. 
Figura 2. Filme à base de amido de mesocarpo de babaçu

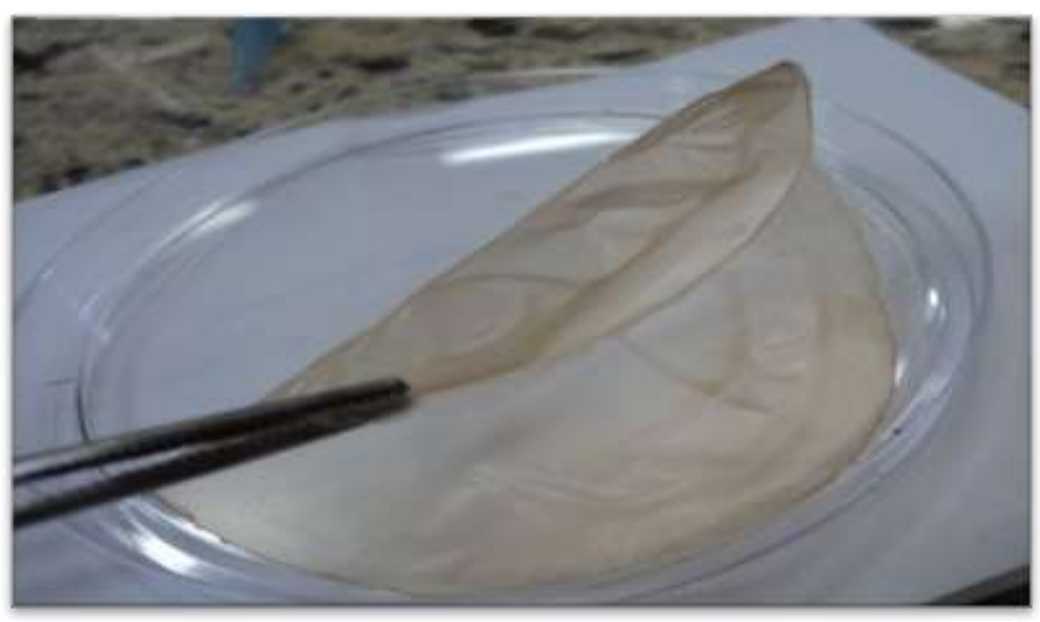

Fonte: Autores (2021).

\section{Caracterização dos filmes}

Dentre os objetivos de um bom curativo para feridas está a capacidade de promover a migração celular, para isso o curativo deve ter uma taxa de transmissão de vapor de água satisfatória, que juntamente com a capacidade de absorção de água irão promover o controle do balanço de fluidos. Os filmes foram avaliados quanto à homogeneidade, espessura, propriedades físicas e mecânicas.

\section{Testes físicos}

A Tabela 1 mostra os resultados das características físicas dos filmes de mesocarpo de babaçu sem e com Aloe vera, avaliando-se a influência desse composto bioativo nas concentrações de 5, 8 e 10\% de Aloe vera em relação à massà base do filme.

Tabela 1. Teor de umidade ( $\omega)$, espessura ( $\delta$ ), solubilidade em água (S) e permeabilidade ao vapor de água (PVA) de filmes de mesocarpo de babaçu e Aloe vera.

\begin{tabular}{lcccc}
\hline Formulações & $\begin{array}{c}\boldsymbol{S} \\
(\boldsymbol{\%}, \mathbf{b . u})\end{array}$ & $\begin{array}{c}\mathbf{S} \\
(\boldsymbol{\%}, \mathbf{b . u})\end{array}$ & $\begin{array}{c}\boldsymbol{\delta} \\
(\mathbf{m m})\end{array}$ & $\begin{array}{c}\text { PVA } \\
\left(\mathbf{g} . \mathbf{m m} / \mathbf{m}^{2} . \mathbf{d i a} \cdot \mathbf{k P a}\right)\end{array}$ \\
\hline FMSAV & $12,38 \pm 1,31^{\mathrm{a}}$ & $23,46 \pm 3,59^{\mathrm{bc}}$ & $0,10 \pm 0,01^{\mathrm{a}}$ & $4,58 \pm 0,15^{\mathrm{b}}$ \\
FMAV5\% & $9,84 \pm 0,99^{\mathrm{a}}$ & $11,73 \pm 1,70^{\mathrm{c}}$ & $0,11 \pm 0,01^{\mathrm{a}}$ & $5,82 \pm 0,80^{\mathrm{b}}$ \\
FMAV8\% & $11,65 \pm 1,10^{\mathrm{a}}$ & $41,85 \pm 8,64^{\mathrm{ab}}$ & $0,12 \pm 0,02^{\mathrm{a}}$ & $8,13 \pm 0,67^{\mathrm{a}}$ \\
FMAV10\% & $13,59 \pm 0,73^{\mathrm{a}}$ & $49,83 \pm 5,95^{\mathrm{a}}$ & $0,12 \pm 0,01^{\mathrm{a}}$ & $8,32 \pm 0,67^{\mathrm{a}}$ \\
\hline
\end{tabular}

As mesmas letras na mesma coluna indicam que não há diferença significativa entre os valores das médias $(p<0,05)$ pelo teste de Tukey. Fonte: Autores (2021).

De acordo com essa tabela, o teor de umidade foi de 12,38\% para o filme sem Aloe vera e para os filmes com Aloe vera aumentou de acordo a concentração desse componente, sendo o menor valor de 9,84\%, para o filme com 5\% de Aloe vera e o maior valor 13,59\% para o filme com 10\% de Aloe vera, o que pode estar relacionado a alta afinidade de Aloe vera com água (Femenia et al., 2003; Pereira et al., 2013). Entretanto, estatisticamente estes valores não diferiram.

A solubilidade em água dos filmes seguiu o mesmo comportamento do teor de umidade, sendo de 23,46\% para o filme sem Aloe vera. A solubilidade tornou-se maior com o aumento na concentração de Aloe vera, de acordo com a Tabela 1, variando 
entre 11,73\% e 49,83\%. Os resultados indicam que os filmes são pouco solúveis após a imersão em água destilada por 24 h, embora tenham aumentado com o aumento na proporção de Aloe vera o que pode ser devido a maior absorção de água. A massa solubilizada foi maior entre as amostras com 5\% e 8\% de Aloe vera ( $\mathrm{p}<0,05)$ e não estatisticamente diferente entre $8 \%$ e $10 \%$ de Aloe vera $(\mathrm{p}>0,05)$, o que pode indicar uma redução da solubilidade a partir dessa concentração.

Conforme ilustrado na Tabela 1 a incorporação de Aloe vera na solução filmogênica não alterou significativamente a espessura dos filmes ( $p>0,05$ ); os valores variaram entre 0,10 e $0,12 \mathrm{~mm}$. Foi observado comportamento parecido nas características de solubilidade e espessura encontradas nos filmes deste trabalho no estudo de Pereira et al. (2013) que avaliaram as características físicas de filmes de hidrogel à base de alginato com diferentes proporções de Aloe vera.

Os resultados de PVA encontrados foram considerados moderados, devido a possíveis falhas microestruturais que permitem a entrada de vapores, sendo 4,58 (g.mm)/( $\mathrm{m}^{2}$.dia.kPa) para à base do filme. Este valor diferente estatisticamente dos filmes com Aloe vera ( $\mathrm{p}<0,05)$, que variaram de 5,82 a 8,32 (g.mm)/( $\mathrm{m}^{2}$.dia.kPa). Entre os filmes com 8 e $10 \%$ de Aloe vera não houve diferença significativa $(\mathrm{p}>0,05)$.

Um fator de grande influência na PVA é o material utilizado na formulação, além disso, condições ambientais, como umidade relativa, temperatura e velocidade do ar local, também podem influenciar no sucesso do curativo (Wu et al., 1995; Maniglia et al., 2019).

O mesocarpo de coco babaçu possui alta capacidade de intumescimento e, quando misturado ao glicerol, cria um sistema plastificante que mantém a umidade da matriz do biofilme controlada. Esse efeito sinérgico está associado ao processo de dispersão do mesocarpo de babaçu no glicerol, o que reduz a disponibilidade de grupos hidroxila para interagir com a água, fator determinante para o teor de umidade característico de cada filme (Da Silva et al., 2019). A presença da Aloe vera parece contribuir para o aumento desse efeito. Mais testes precisam ser realizados para comprovar essas interações.

\section{Propriedades mecânicas}

Na Tabela 2 estão apresentados dos valores de resistência à tração (TR), tensão de ruptura (e), módulo de Youg (E), de filmes de mesocarpo de babaçu e Aloe vera.

O conhecimento das propriedades mecânicas de estruturas poliméricas é importante para avaliar a qualidade dos filmes destinados ao uso como curativo. Os parâmetros mecânicos, químicos e estruturais e os parâmentos externos dos polímeros podem influenciar diretamente suas respostas mecânicas (Paixão et al., 2019). Das diversas características químicas e estruturais dos polímeros, algumas que alteram seu comportamento mecânico são cristalinidade, a presença de grupos polares e ligações cruzadas. Os seguintes parâmetros externos também podem influenciar o comportamento mecânico dos polímeros: a presença de um plastificante, elastômeros e o reforço com fibras, entre outros (da Silva Raposo et al., 2020). A Tabela 2 mostra que a tensão de ruptura que variaram de 1,10 a 2,35 MPa, não diferindo estatisticamente ao nível de 95\% de confiança pelo teste de Tukey.

Entretanto, a maior tensão de ruptura pode estar relacionada ao número de grupos polares presentes no Aloe vera, que aumentam com o aumento na concentração. Isso resultou em uma interação maior entre as cadeias por meio de ligações secundárias, consequentemente aumentando a resistência mecânica do filme, ou seja, a Aloe vera usada nesta pesquisa poderia ter uma massa molar baixa e, portanto, tornam-se miscíveis com o polímero, que, quando incorporado, interagiu e ocupou fisicamente todo o espaço entre as cadeias poliméricas, tornando as interações entre elas difíceis e assim aumentando tensão de ruptura. No entanto, os resultados obtidos nesta pesquisa, foram inferiores aos obtidos em outros estudos utilizando mesocarpo de coco babaçu (Da Silva et al., 2019; da Silva Raposo et al., 2020; Lopes et al., 2020). 
Tabela 2. Resistência à tração (TR), tensão de ruptura (e), módulo de Youg (E), de filmes de mesocarpo de babaçu e Aloe vera.

\begin{tabular}{lccc}
\hline Formulações & TR & $\mathrm{e}$ & $\mathrm{E}$ \\
\hline FMSAV & $(\mathrm{MPa})$ & $(\%)$ & $(\mathrm{MPa})$ \\
FMAV5\% & $1,10 \pm 0,27$ & $14,98 \pm 17,86000$ & $105,54 \pm 95,42$ \\
FMAV8\% & $1,87 \pm 0,15$ & $1,21 \pm 0,34$ & $165,78 \pm 30,58$ \\
FMAV10\% & $1,94 \pm 1,12$ & $1,39 \pm 0,25$ & $197,09 \pm 105,27$ \\
\hline
\end{tabular}

As mesmas letras na mesma coluna indicam que não há diferença significativa entre os valores das médias $(p<0,05)$ pelo teste de Tukey. Fonte: Autores (2021).

Os resultados obtidos demonstram que a adição de Aloe vera não alterou significativamente o alongamento na ruptura e módulo de Young dos filmes, conforme mostrado na Tabela 2, mesmo com a diminuição nos teores de amido e glicerol dentro dos filmes.

Segundo Paixão et al. (2019), o alongamento na ruptura é a porcentagem de mudança no comprimento do filme quando o filme é quebrado após um certo nível de força é aplicada. Como contrapartida, os filmes sem Aloe vera apresentaram valor maior para alongamento na ruptura, quando comparados com os que continham essa substância. Outro fato que pode ter ocorrido, é que com a adição do Aloe vera pode ter ocasionado a desplastificação do polímero (neste caso glicerol) fazendo com que o alongamento diminuísse.

A presença do glicerol nos filmes tem influência significativa em suas propriedades mecânicas, causando redução na rigidez, diminuindo a tensão de ruptura e aumentando o alongamento na ruptura. Esse comportamento pode ser explicado pelo efeito plastificante do glicerol, que melhora o volume livre entre as cadeias poliméricas, reduzindo as interações polímeropolímero e aumentando a mobilidade das cadeias poliméricas (Pereira et al., 2013).

Considerando as espessuras médias e as formulações utilizadas na fabricação dos filmes os resultados demonstram que os filmes casting de mesocarpo de babaçu com Aloe vera são promissores para o desenvolvimento de curativos para proteção de feridas e absorção do exsudato. Mais testes precisam ser realizados para confirmar essas características.

\section{Efeito citotóxico em macrófagos RAW 264.7 da solução filmogênica}

Para as formulações testadas houve diminuição significativa da viabilidade dos macrófagos apenas na maior concentração dà base do filme mais Aloe Vera 10 \% ( $p$ < 0.05) (Figura 3). Como nenhuma das concentrações teve citotoxicidade acima de $50 \%$, a concentração citotóxica sobre $50 \%$ dos macrófagos $\left(\mathrm{CC}_{50}\right)$ foi considerada como $\mathrm{CC}_{50}>10 \%$. A elucidação sobre o mecanismo de ação desta matriz polimérica sobre os macrófagos RAW 264.7 necessita de maiores investigações. 
Figura 3. Efeito citotóxico em macrófagos RAW 264.7 após 72 h de tratamento.
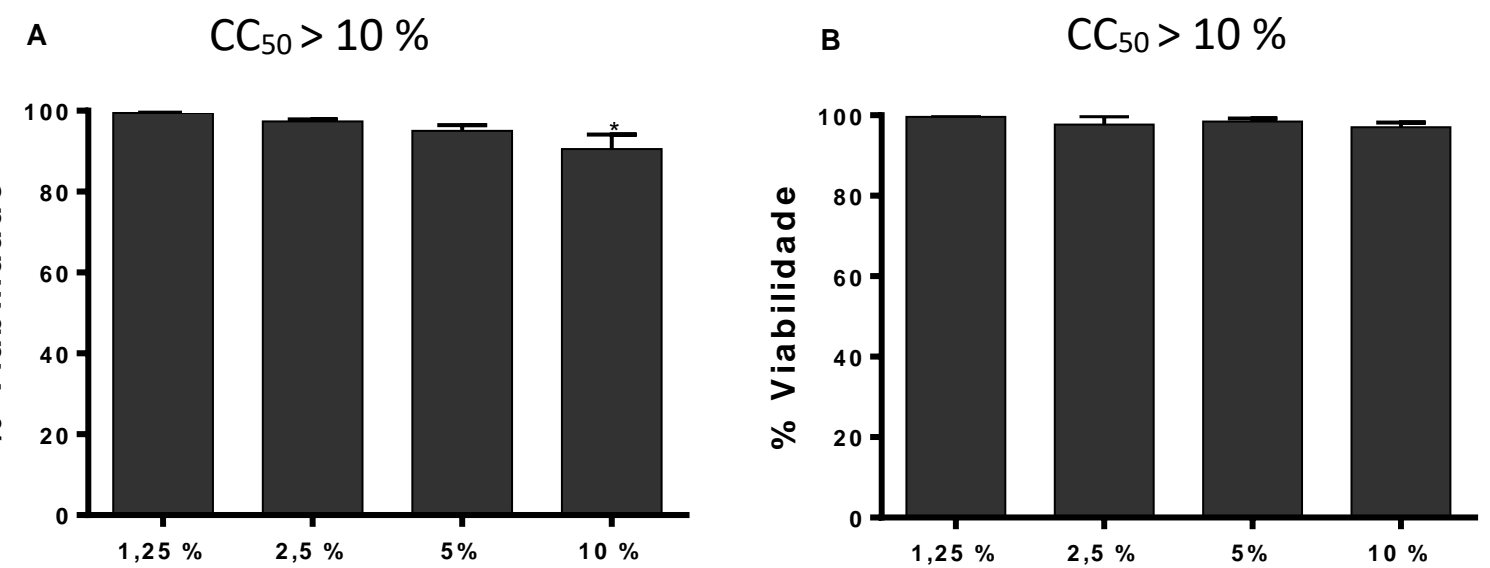

$\mathrm{A}$ - Base do filme + Aloe vera $\mathrm{B}$ - base do filme. Os macrófagos RAW 264.7 foram incubados por $72 \mathrm{~h}$ à temperatura de $37^{\circ} \mathrm{C}$ em estufa a $5 \%$ de $\mathrm{CO}_{2}$, na ausência e na presença de diferentes concentrações dà base, e, posteriormente, a viabilidade celular foi avaliada pelo método de redução do 3-[4,5-dimetiltiazol-2-il]-2,5- difeniltetrazolium brometo (MTT). O gráfico representa a média \pm erro padrão da média de três experimentos independentes realizados em triplicata, considerando o controle (DMSO 0,5\% em meio DMEM completo) como 100\% de viabilidade. One-way ANOVA seguido pelo pós-teste de Tukey foi realizado para comparação entre os grupos, sendo *p<0,05 ao controle. Fonte: Autores (2021).

Os resultados obtidos concordam com os estudos de Carvalho, et al. (2018), que testaram o extrato de Aloe vera o qual apresentou baixa citotoxicidade em macrófagos. As propriedades antiinflamatórias dessa planta bloqueiam a inflamação e estimulam o crescimento de fibroblastos, acelerando o processo de cicatrização.

De Sousa Leal et al. (2018) identificaram uma baixa citotoxicidade em macrófagos peritoneais de camundongos Balb de filmes de mesocarpo de Orbignya sp. e carboximetilcelulose para aplicação como matriz de liberação de fármacos.

Os macrófagos desempenham um papel fundamental no início da inflamação e são metabolicamente ativos, sendo distribuídos no tecido conjuntivo o que influencia as funções locais e sistêmicas no reparo de lesões (Oishi \& Manabe, 2018). Valores elevados de viabilidade celular de macrófagos em relação aos grupos controle são uma evidência importante que demonstra a capacidade da formulação de se associar a membranas biológicas (Santos et al., 2021).

\section{Atividade hemolítica da solução filmogênica}

A avaliação da citotoxicidade hemolítica é um dos métodos mais eficientes que analisa a hemólise provocada por uma substância (extrato de plantas medicinais e suas formulações) quando a mesma é incubada com uma suspensão padronizada de hemácias (de Haro Moreno et al., 2018).

O uso de formulações em feridas pode promover acesso à corrente sanguínea, causando efeitos deletérios aos eritrócitos, portanto é importante avaliação do percentual de hemólise sanguínea para o desenvolvimento de novos agentes terapêuticos (Maciel et al., 2002; Santos et al., 2020).

A Figura 4 mostra o perfil de hemólise das formulações da solução de mesocarpo em relação a concentração de Aloe Vera, nota-se que para a formulação com $0 \%$ de Aloe vera houve 10,27\% \pm 0,2309 de hemólise, a de 5\% de Aloe vera apresentou $10,47 \% \pm 0,1155$, a de $8 \%$ de Aloe vera foi de $11,67 \% \pm 0,1155$, enquanto que o produto com $10 \%$ de Aloe vera foi de $12,07 \%$ $\pm 0,3055$. O controle positivo da hemólise foi de $82,27 \% \pm 2,301$. O controle positivo foi considerando estaticamente significativo com $p=0,0002$. Observa-se que não houve diferença significativa no percentual de rompimento das hemácias com o aumento da concentração de Aloe vera na solução filmogênica.

Os resultados obtidos sugerem que o uso dessas formulações é seguro, baseado na comparação com o estudo de Dias et al. (2018) que avaliaram o efeito citotóxico do gel de Aloe vera, em que não houve taxa de hemólise significativa pelo teste de 
fragilidade osmótica que evidenciasse risco tóxico, concluindo que a substância pode ser utilizada de forma segura em tratamento tópico de queimaduras e com atividade bacteriostática para os principais microrganismos responsáveis por infecção em pacientes queimados.

Figura 4. Efeito da solução filmogênica sobre hemácias humanas (atividade hemolítica) avaliado nas concentrações de $0,5,8 \mathrm{e}$ $10 \%$ de Aloe vera em relação a massa base dos filmes.

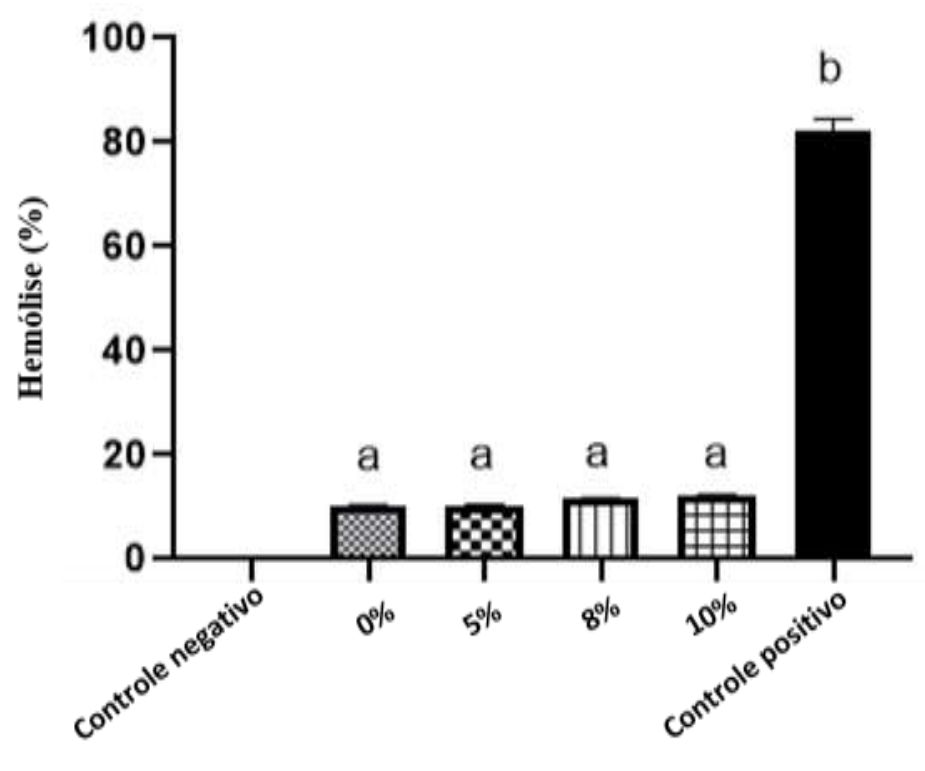

Solução Filmogênica

Letras diferentes nos dados foram considerados estatisticamente significativos de acordo com o teste de Tukey usando o ANOVA. Fonte: Autores (2021).

A segurança do uso do extrato aquoso do mesocarpo de babaçu foi testada por De Araújo et al. (2013) que em seus ensaios de hemólise mostraram que a concentração de hemoglobina foi maior na cultura de hemácias tratadas com o extrato na concentração de $40 \mathrm{mg} / \mathrm{mL}$ do que quando tratadas com o controle, foi ainda observado um efeito imunomodulador apresentando uma participação no sistema complemento, possivelmente através da via das lectinas.

\section{Conclusão}

Os filmes apresentaram superfície lisa e boa maleabilidade, são autossustentados e estáveis. Foram avaliadas as características físicas dos filmes, que apresentaram teor de umidade de 12,38\% para o FMSA, 9,84\% para o FMAV 5\%, 11,65\% para o FMAV 8\% e 13,59\% para o FMAV 10\%, não houve diferença estatística entre esses valores. A solubilidade para o FMSA é maior que FMAV, dentre os FMAV a solubilidade é diretamente proporcional a concentração. Os filmes são pouco solúveis após a imersão em água destilada por 24 horas. A espessura dos filmes não foi alterada significativamente com a incorporação de Aloe vera. Os resultados de PVA foram considerados moderados sendo 4,58 (g.mm)/(m2.dia.kPa) para o FMSA. Este valor diferente estatisticamente dos filmes com Aloe vera ( $\mathrm{p}<0,05)$, que variaram de 5,82 a 8,32 (g.mm)/(m2.dia.kPa), entre os filmes com 8 e $10 \%$ de Aloe vera não houve diferença significativa $(p>0,05)$.

As características mecânicas demonstraram tensão de ruptura variando de 1,10 a 2,35 MPa, sendo maior de acordo com o aumento na concentração de Aloe vera, o que pode estar relacionado com o aumento de grupos polares resultando em uma maior interação entre as cadeias por meio de ligações secundárias, consequentemente aumentando a resistência mecânica do filme. Esses resultados foram inferiores aos obtidos em outros estudos utilizando mesocarpo do coco babaçu. A adição de Aloe 
vera não alterou significativamente o alongamento na ruptura e módulo de Young dos filmes.

A citotoxicidade da solução filmogênica foi testada em macrófagos murinos da linhagem RAW 264.7 e em hemácias humanas, sendo considerada segura para a aplicação em superfícies de feridas.

As características observadas nas formulações dos filmes testados são promissoras no desenvolvimento de curativos para proteção de feridas e absorção do exsudato. Mais testes precisam ser realizados para confirmar essas características.

\section{Agradecimentos}

Agradecemos à CNPq, CAPES e FAPEMA pelo suporte financeiro e ao Laboratório de Engenharia de Produtos e Processos em Biorecursos da Universidade Federal do Maranhão pelo apoio, infraestrutura disponibilizada para a pesquisa, análise e discussão de dados.

\section{Referências}

AOAC, Official Methods of Analysis AOAC (2007). International, Gaithersburg.

Ashraf, R., Sofi, H. S., Malik, A., Beigh, M. A., Hamid, R., \& Sheikh, F. A. (2019). Recent trends in the fabrication of starch nanofibers: Electrospinning and non-electrospinning routes and their applications in biotechnology. Applied biochemistry and biotechnology, 187(1), 47-74.

ASTM International (2016) ASTM E96/E96M-16. In Standard, test methods of water vapor transmission of materials. West Conshohocken.

ASTM International ASTM D-882 (2012). Standard test method for tensile properties in thin flms sheeting. In ASTMD882-18. West Conshohocken.

Azevedo, A. P. S., Farias, J. C., Costa, G. C., Ferreira, S. C., Aragao-Filho, W. C., Sousa, P. R., Pinheiro, M. T., Maciel, M. C., Silva, L. A., \& Lopes, A. S. (2007). Anti-thrombotic effect of chronic oral treatment with Orbignya phalerata Mart. Journal of Ethnopharmacology, 111(1), 155-159.

Batista, C. P., Torres, O. J. M., Matias, J. E. F., Moreira, A. T. R., Colman, D., Lima, J. H. F. de, Macri, M. M., Rauen Jr, R. J., Ferreira, L. M., \& Freitas, A. C. T. de. (2006). Effect of watery extract of Orbignya phalerata (babassu) in the gastric healing in rats: Morfologic and tensiometric study. Acta Cirurgica Brasileira, $21,26-32$.

Brito, M. C. A. (2015). Farmacovigilância em fitoterapia: controle de qualidade do mesocarpo de Attalea speciosa Mart. ex Spreng. (Babaçu). Programa de Pósgraduação em Ciências da Saúde. Dissertação (Mestrado). Universidade Federal do Maranhão, São Luís, 112p.

Caetano, N., Saraiva, A., Pereira, R., Carvalho, D., Pimentel, M. C. B., \& Maia, M. B. S. (2002). Determinação de atividade antimicrobiana de extratos de plantas de uso popular como antiflamatório. Revista Brasileira de farmacognosia, 12, 132-135.

Carvalho, N. C., Guedes, S. A. G., Albuquerque-Júnior, R. L. C., de Albuquerque, D. S., de Souza Araújo, A. A., Paranhos, L. R., Camargo, S. E. A., \& Ribeiro, M. A. G. (2018). Analysis of Aloe vera cytotoxicity and genotoxicity associated with endodontic medication and laser photobiomodulation. Journal of Photochemistry and Photobiology B: Biology, 178, 348-354.

Da Silva, D. C., Lopes, I. A., Da Silva, L. J. S., Lima, M. F., Barros Filho, A. K. D., Villa-Vélez, H. A., \& Santana, A. A. (2019). Physical properties of films based on pectin and babassu coconut mesocarp. International journal of biological macromolecules, 130, 419-428.

da Silva Raposo, A. K., Paixão, L. C., Rocha, A. A., Lopes, I. A., Santos, G. A. S., Ribeiro, G. A. C., de Menezes, A. S., Barros Filho, A. K. D., \& Santana, A. A. (2020). Characterization of Biodegradable Films Produced from Mixtures of Alginate, Starch and Babassu Fibers. Journal of Polymers and the Environment, $1-15$.

De Almeida, R. R., Lacerda, L. G., Murakami, F. S., Bannach, G., Demiate, I. M., Soccol, C. R., da Silva Carvalho Filho, M. A., \& Schnitzler, E. (2011). Thermal analysis as a screening technique for the characterization of babassu flour and its solid fractions after acid and enzymatic hydrolysis. Thermochimica Acta, 519(1-2), 50-54.

de Araújo, E. M. M., de Almeida, C. S. C., Junior, J. M. F. G., \& do Nascimento, F. R. F. (2013). Ativação in vitro do sistema complemento como mecanismo imunomodulador induzido pelo mesocarpo de babaçu. Revista de Ciências da Saúde, 15(1).

de Haro Moreno, A., Possebon, L., Sant'ana, M., Souza, H. R., Pilon, M. M. I., \& Girol, A. P. (2018). Avaliação da atividade antimicrobiana e citotoxicidade hemolítica em diferentes extratos vegetais. Arquivos de Ciências Da Saúde, 25(1), 11-12.

de Sousa Leal, A., de Araújo, R., Souza, G. R., Lopes, G. L. N., Pereira, S. T., de Moraes Alves, M. M., Barreto, H. M., Carvalho, A. L. M., Ferreira, P. M. P., \& Silva, D. (2018). In vitro bioactivity and cytotoxicity of films based on mesocarp of Orbignya sp. And carboxymethylcellulose as a tannic acid release matrix. Carbohydrate polymers, 201, 113-121.

Dias, J. L., Lacerda, G. E., Cabral, J. B., Moreira, J. F., Dias, T., \& do Nascimento, G. N. L. (2018). Propriedade antimicrobiana e potencial citotóxico in vitro do gel de Aloe vera: Uma discussão sobre o uso em queimaduras. Scientia Plena, 14(4)

Eskandarinia, A., Kefayat, A., Rafienia, M., Agheb, M., Navid, S., \& Ebrahimpour, K. (2019). Cornstarch-based wound dressing incorporated with hyaluronic acid and propolis: In vitro and in vivo studies. Carbohydrate polymers, 216, 25-35. 
Femenia, A., García-Pascual, P., Simal, S., \& Rosselló, C. (2003). Effects of heat treatment and dehydration on bioactive polysaccharide acemannan and cell wall polymers from Aloe barbadensis Miller. Carbohydrate polymers, 51(4), 397-405.

Fortes, T. S., Azevedo, A. P. S., Barroqueiro, E. S., Pinheiro, M. T., Guerra, R. N. M., Reis, A. S., Fialho, E. M. S., Assunção, A. K. M., \& Nascimento, F. R. F. (2009). Desenvolvimento do Tumor de Ehrlich em Camundongos após incubação in vitro com mesocarpo de babaçu. Revista de Ciências da Saúde, 11, 1118 .

Jithendra, P., Rajam, A. M., Kalaivani, T., Mandal, A. B., \& Rose, C. (2013). Preparation and characterization of aloe vera blended collagen-chitosan composite scaffold for tissue engineering applications. ACS applied materials \& interfaces, 5(15), 7291-7298.

Khan, A. W., Kotta, S., Ansari, S. H., Sharma, R. K., Kumar, A., \& Ali, J. (2013). Formulation development, optimization and evaluation of aloe vera gel for wound healing. Pharmacognosy magazine, 9(Suppl 1), S6.

Lima, L. L., Bierhalz, A. C. K., \& Moraes, Â. M. (2020). Influence of the chemical composition and structure design of electrospun matrices on the release kinetics of Aloe vera extract rich in aloin. Polymer Degradation and Stability, 179, 109233.

Lopes, I. A., Paixão, L. C., da Silva, L. J. S., Rocha, A. A., Barros Filho, A. K. D., \& Santana, A. A. (2020). Elaboration and characterization of biopolymer films with alginate and babassu coconut mesocarp. Carbohydrate polymers, 234, 115747.

Maciel, M. A. M., Pinto, A. C., Veiga Jr, V. F., Grynberg, N. F., \& Echevarria, A. (2002). Plantas medicinais: A necessidade de estudos multidisciplinares. Química nova, 25(3), 429-438.

Maniglia, B. C., Tessaro, L., Ramos, A. P., \& Tapia-Blácido, D. R. (2019). Which plasticizer is suitable for films based on babassu starch isolated by different methods? Food Hydrocolloids, 89, 143-152.

Nascimento, F. R., Barroqueiro, E. S., Azevedo, A. P. S., Lopes, A. S., Ferreira, S. C., Silva, L. A., Maciel, M. C., Rodriguez, D., \& Guerra, R. N. (2006). Macrophage activation induced by Orbignya phalerata Mart. Journal of ethnopharmacology, 103(1), 53-58.

Oishi, Y., \& Manabe, I. (2018). Macrophages in inflammation, repair and regeneration. International immunology, 30(11), 511-528.

Oliveira, D. R. de. (2011). Estudo etnofarmacognóstico da saracuramirá (Ampelozizyphus amazonicus Ducke), uma planta medicinal usada por comunidades quilombolas do Município de Oriximiná-PA, Brasil. Acta amaz, 383-392.

Oryan, A., T Naeini, A., Nikahval, B., \& Gorjia, E. (2010). Effect of aqueous extract of Aloe vera on experimental cutaneous wound healing in rat. Veterinarski arhiv, 80(4), 509-522.

Paixão, L. C., Lopes, I. A., Barros Filho, A. K. D., \& Santana, A. A. (2019). Alginate biofilms plasticized with hydrophilic and hydrophobic plasticizers for application in food packaging. Journal of Applied Polymer Science, 136(48), 48263.

Pan, L., Zhang, X., \& Gao, Q. (2021). Effects and mechanisms of histatins as novel skin wound-healing agents. Journal of Tissue Viability.

Pathalamuthu, P., Siddharthan, A., Giridev, V. R., Victoria, V., Thangam, R., Sivasubramanian, S., Savariar, V., \& Hemamalini, T. (2019). Enhanced performance of Aloe vera incorporated chitosan-polyethylene oxide electrospun wound scaffold produced using novel Spirograph based collector assembly. International journal of biological macromolecules, 140, 808-824.

Pereira, R., Mendes, A., \& Bártolo, P. (2013). Alginate/Aloe vera hydrogel films for biomedical applications. Procedia CIRP, 5, $210-215$.

Rennó, M. N., Barbosa, G. M., Zancan, P., Veiga, V. F., Alviano, C. S., Sola-Penna, M., Menezes, F. S., \& Holandino, C. (2008). Crude ethanol extract from babassu (Orbignya speciosa): Cytotoxicity on tumoral and non-tumoral cell lines. Anais da Academia Brasileira de Ciencias, 80(3), 467-476.

Rezler, R., \& Poliszko, S. (2010). Temperature dependence of starch gel rheological properties. Food Hydrocolloids, 24(6-7), 570-577.

Rodrigues, R. R. L., Nunes, T. A. L., de Araújo, A. R., Marinho Filho, J. D. B., da Silva, M. V., de Amorim Carvalho, F. A., Pessoa, O. D. L., Freitas, H. P. S., da Franca Rodrigues, K. A., \& Araújo, A. J. (2021). Antileishmanial activity of cordiaquinone E towards Leishmania (Leishmania) amazonensis. International Immunopharmacology, 90, 107124.

Santos, A. P. (2009). Extração e caracterização do amido do fruto-do-lobo (Solanum lycocarpum St. Hil) e elaboração de filmes biodegradáveis. Mestrado (Dissertação). Anápolis.

Santos, D. C. P., Gomes, P. D. B., Leite, D. S., Nova, B. G. V., Alves, M. B., Viana, P. R. S., Rodrigues, J. A., de Mattos-Guaraldi, A. L., Sabbadini, P. S., \& Firmo, W. da C. A. (2020). Bioprospecção das atividades antioxidante, antibacteriana e antibiofilme contra Corynebacterium ulcerans e toxicidade de Stryphnodendron coriaceum Benth. Scientia Plena, 16(10).

Santos, D. S., Morais, J. A. V., Vanderlei, Í. A., Santos, A. S., Azevedo, R. B., Muehlmann, L. A., Júnior, O. R., Mortari, M. R., da Silva, J. R., \& da Silva, S. W. (2021). Oral delivery of fish oil in oil-in-water nanoemulsion: Development, colloidal stability and modulatory effect on in vivo inflammatory induction in mice. Biomedicine \& Pharmacotherapy, 133, 110980.

Silva, S. S., Popa, E. G., Gomes, M. E., Cerqueira, M., Marques, A. P., Caridade, S. G., Teixeira, P., Sousa, C., Mano, J. F., \& Reis, R. L. (2013). An investigation of the potential application of chitosan/aloe-based membranes for regenerative medicine. Acta biomaterialia, 9(6), 6790-6797.

Surjushe, A., Vasani, R., \& Saple, D. G. (2008). Aloe vera: A short review. Indian journal of dermatology, 53(4), 163.

Tapia-Blácido, D. R., do Amaral Sobral, P. J., \& Menegalli, F. C. (2011). Optimization of amaranth flour films plasticized with glycerol and sorbitol by multiresponse analysis. LWT-Food Science and Technology, 44(8), 1731-1738.

Teixeira, M. A. (2008). Babassu—A new approach for an ancient Brazilian biomass. Biomass and Bioenergy, 32(9), 857-864. 
Research, Society and Development, v. 10, n. 2, e30310212520, 2021

(CC BY 4.0) | ISSN 2525-3409 | DOI: http://dx.doi.org/10.33448/rsd-v10i2.12520

Tummalapalli, M., Berthet, M., Verrier, B., Deopura, B. L., Alam, M. S., \& Gupta, B. (2016). Composite wound dressings of pectin and gelatin with aloe vera and curcumin as bioactive agents. International journal of biological macromolecules, 82, 104-113.

Wu, P., Fisher, A. C., Foo, P. P., Queen, D., \& Gaylor, J. D. S. (1995). In vitro assessment of water vapour transmission of synthetic wound dressings. Biomaterials, 16(3), 171-175.

Yang, Z.-G., Sun, H.-X., \& Fang, W.-H. (2005). Haemolytic activities and adjuvant effect of Astragalus membranaceus saponins (AMS) on the immune responses to ovalbumin in mice. Vaccine, 23(44), 5196-5203. 